\title{
AN UNCOUPLING PROCEDURE FOR A CLASS OF COUPLED LINEAR PARTIAL DIFFERENTIAL EQUATIONS
}

\author{
A. MCNABB ${ }^{1}$
}

(Received 14 June 1983; revised 21 December 1983)

\begin{abstract}
A Fredholm operator exists which maps the solutions of a system of linear partial differential equations of the form $\partial u / \partial t=D L u+A u$ coupled by a matrix $A$ onto those solutions of a similar system coupled by a matrix $B$ which have the same initial values. The kernels of this operator satisfy a hyperbolic system of equations. Since these equations are independent of the linear partial differential operator $L$, the same operator serves as a mapping for a large class of equations. If $B$ is chosen diagonal, the solutions of a coupled system with matrix $A$ may be obtained from the uncoupled system with matrix $B$.
\end{abstract}

\section{Introduction}

Hill [3] considered the coupled system

$$
\begin{array}{ll}
\frac{\partial u_{1}}{\partial t}=d_{1} L u_{1}-a_{1} u_{1}+b_{1} u_{2}, & u_{1}(x, 0)=f_{1}(x), \\
\frac{\partial u_{2}}{\partial t}=d_{2} L u_{2}+b_{2} u_{1}-a_{2} u_{2}, & u_{2}(x, 0)=f_{2}(x),
\end{array}
$$

where $L$ denotes a linear constant coefficient differential operator involving spatial derivatives only. He showed that if $h_{1}, h_{2}$ are solutions of the uncoupled system,

$$
\frac{\partial h_{i}}{\partial t}=L h_{i}, \quad h_{i}(x, 0)=f_{i}(x)
$$

\footnotetext{
${ }^{1}$ Applied Mathematics Divsion, Department of Scientific and Industrial Research, Wellington, New Zealand.

() Copyright Australian Mathematical Society 1985, Serial-fee code 0334-2700/85
} 
then a solution of the coupled system is given by

$$
\begin{aligned}
& u_{1}(x, t)=e^{-a_{1} t} h_{1}\left(x, d_{1} t\right)+\frac{e^{\lambda t}}{\left(d_{1}-d_{2}\right)} \int_{d_{2} t}^{d_{1} t} e^{-\mu \xi} \\
& \times\left[\left\{\frac{b_{1} b_{2}\left(\xi-d_{2} t\right)}{\left(d_{1} t-\xi\right)}\right\}^{1 / 2} I_{1}(\eta) h_{1}(x, \xi)+b_{1} I_{0}(\eta) h_{2}(x, \xi)\right] d \xi, \\
& u_{2}(x, t)=e^{-a_{2} t h_{2}\left(x, d_{2} t\right)+\frac{e^{\lambda t}}{\left(d_{1}-d_{2}\right)} \int_{d_{2} t}^{d_{1} t} e^{-\mu \xi}}
\end{aligned}
$$$$
\times\left[\left\{\frac{b_{1} b_{2}\left(d_{1} t-\xi\right)}{\left(\xi-d_{2} t\right)}\right\}^{1 / 2} I_{1}(\eta) h_{2}(x, \xi)+b_{2} I_{0}(\eta) h_{1}(x, \xi)\right] d \xi
$$

where

$$
\begin{gathered}
\lambda=\left(a_{1} d_{2}-a_{2} d_{1}\right) /\left(d_{1}-d_{2}\right), \quad \mu=\left(a_{1}-a_{2}\right) /\left(d_{1}-d_{2}\right), \\
\eta=2\left[b_{1} b_{2}\left(d_{1} t-\xi\right)\left(\xi-d_{2} t\right)\right]^{1 / 2} /\left(d_{1}-d_{2}\right),
\end{gathered}
$$

and $I_{0}$ and $I_{1}$ are modified Bessel functions.

A matrix formulation and generalisation of this two variable case is studied in this paper. Suppose $h$ is a mapping from $R^{m+1}$ to $R^{N}$ satisfying

$$
\begin{aligned}
& \frac{\partial h}{\partial t}=D L h \text { in }\left\{(x, t): x \in \Omega \text { in } R^{m}, 0<t<T\right\}, \\
& h(x, 0)=f(x), \quad x \in \Omega,
\end{aligned}
$$

where $D$ is a constant nonsingular diagonal matrix and the operator $L$ commutes with any $B \in S_{N}\left(0, T_{0}\right)$, the set of regulated mappings from $(0, T)$ into $N \times N$ matrices with real $i j$ th element $B_{i j}[1]$. We show that, given $A \in S_{N}\left(0, T_{0}\right)$, there is a $J \in S_{N}\left(0, T_{0}\right)$, and a pair of kernels $k^{+}(t, s), k^{-}(t, s)$ such that

$$
\begin{aligned}
u(x, t) & =J h(x, t)+\int_{0}^{t} k^{+}(t, s) h(x, s) d s+\int_{t}^{T_{0}} k^{-}(t, s) h(x, s) d s \\
& \equiv(J+K) h(x, t) .
\end{aligned}
$$

is a solution of the equations

$$
\frac{\partial u}{\partial t}=D L u+A u, \quad u(x, 0)=f(x)
$$

If it is assumed such an operator $J+K$ exists, then equations (1.1), (1.2) and (1.3) imply $J$ must satisfy an ordinary differential equation and commute with $D$, while $k^{+}$and $k^{-}$satisfy a hyperbolic system of equations.

In Section 2 we describe these equations and show they have a unique solution dependent only on $D$ and $A$, and that the operator $J+K$ so constructed does indeed map solutions of (1.1) into solutions of (1.3). This operator $J+K$ is shown to be invertible on the space of regulated functions on $\left(0, T_{0}\right)$ with an 
inverse $J^{-1}-H$ of similar form with kernels $h^{+}, h^{-}$satisfying a hyperbolic system of equations in a sense adjoint to those for $k^{+}, k^{-}$.

In Section 3 the hyperbolic systems are solved for the two-variable case and we obtain the results given by Hill [3]. Results for the nonhomogeneous problem are given in Section 4.

\section{Fredholm operator and its inverse for homogeneous problem}

Let $\Omega \subset R^{m}$ denote a bounded domain and $G$ the region $\{(x, t): x \in \Omega$, $\left.0<t<T(x) \leqslant T_{0}\right\}$. Suppose $v(x, t)\left(: G \rightarrow R^{N}\right)$ is a solution of the linear partial differential equation,

$$
\frac{\partial v}{\partial t}=D L v \quad \text { in } G, \quad v(x, 0)=f(x) \text { in } \Omega,
$$

where $D$ is a constant, nonsingular, diagonal matrix with diagonal elements $d_{1} \geqslant d_{2} \geqslant \cdots \geqslant d_{N}$, and the operator $L$ satisfies the commutative relation

$$
L B=B L
$$

for all $B \in S_{N}\left(0, T_{0}\right)$, the set of regulated mappings from $\left(0, T_{0}\right)$ into $N \times N$ matrices.

THEOREM 1. Given a bounded matrix $A \subset S_{N}\left(0, T_{0}\right)$ with $i$ jth element denoted by $A_{i j}$, let

(1) $A^{0} \in S_{N}$ be such that $A_{i j}^{0}=A_{i j}$ when $d_{i}=d_{j}$ and $A_{i j}^{0}=0$ otherwise;

(2) $J \in S_{N}$ be the solution of the differential equation

$$
\frac{d J}{d t}=A^{0} J \text { in }\left(0, T_{0}\right), \quad J(0)=I,
$$

where $I$ is the unit matrix in $S_{N}$.

Then there is a unique pair of functions

$$
\begin{aligned}
& k^{+}(t, s):\left\{(t, s): 0 \leqslant t \leqslant T_{0}, 0 \leqslant s \leqslant t\right\} \rightarrow N \times N \text { matrices, } \\
& k^{-}(t, s):\left\{(t, s): 0 \leqslant s \leqslant T_{0}, 0 \leqslant t \leqslant s\right\} \rightarrow N \times N \text { matrices }
\end{aligned}
$$

which satisfy the hyperbolic equations

$$
\begin{aligned}
& \frac{\partial k^{+}}{\partial t}+D \frac{\partial k^{+}}{\partial s} D^{-1}=A k^{+} \quad \text { in } 0 \leqslant t \leqslant T_{0}, 0 \leqslant s \leqslant t, \\
& \frac{\partial k^{-}}{\partial t}+D \frac{\partial k^{-}}{\partial s} D^{-1}=A k^{-} \text {in } 0 \leqslant s \leqslant T_{0}, 0 \leqslant t \leqslant s,
\end{aligned}
$$

and boundary conditions

$$
\begin{gathered}
k^{+}(t, 0)=k^{-}(0, s)=0, \\
{\left[k^{+}(t, t)-k^{-}(t, t)\right]-D\left[k^{+}(t, t)-k^{-}(t, t)\right] D^{-1}=\left(A-A^{0}\right) J(t)}
\end{gathered}
$$


and the function $u(x, t): G \rightarrow R^{N}$ defined by

$$
\begin{aligned}
u(x, t) & =J(t) v(x, t)+\int_{0}^{t} k^{+}(t, s) v(x, s) d s+\int_{t}^{T_{0}} k^{-}(t, s) v(x, s) d s \\
& \equiv(J+K) v(x, t)
\end{aligned}
$$

is a solution of the differential equation

$$
\frac{\partial u}{\partial t}=D L u+A u \text { in } G, \quad u(x, 0)=f(x)
$$

for $0<t<\min _{i, j}\left(d_{i} / d_{j}\right) T_{0}$.

Proof. The existence proof for $k^{ \pm}(t, s)$ follows classical lines [2] and is merely sketched here. Functions $k^{ \pm}(t, s)$ satisfying equations (2.3), (2.4) can be constructed iteratively as follows. Let

$$
k_{i j}(t, s)= \begin{cases}k_{i j}^{+}(t, s) & \text { in } 0 \leqslant t \leqslant T_{0}, 0 \leqslant s<t \\ k_{i j}^{-}(t, s) & \text { in } 0 \leqslant s \leqslant T_{0}, 0 \leqslant t<s,\end{cases}
$$

and $t=\tau, s=\phi+\left(d_{i} / d_{j}\right) \tau$, so that

$$
\frac{\partial k_{i j}}{\partial \tau}(\tau, \phi)=\sum_{\alpha=1}^{N} A_{i \alpha} k_{\alpha j}(\tau, \phi)
$$

except on $\tau=\phi+\left(d_{i} / d_{j}\right) \tau$.

By integrating this equation along characteristics $\phi=$ constant and using the boundary conditions (2.4), we find: when $i<j$ and $d_{i}>d_{j}$,

$$
\begin{gathered}
s>\frac{d_{i}}{d_{j}} t, k_{i j}(t, s)=\sum_{\alpha=1}^{N} \int_{0}^{t} A_{i \alpha} k_{\alpha j}\left(\theta, s-\frac{d_{i}}{d_{j}}(t-\theta)\right) d \theta \\
t<s<\frac{d_{i}}{d_{j}} t, k_{i j}(t, s)=\frac{d_{j}}{d_{j}-d_{i}} \sum_{\alpha=1}^{N}\left(A_{i \alpha}-A_{i \alpha}^{0}\right) J_{\alpha j}\left(\frac{d_{i} t-d_{j} s}{d_{i}-d_{j}}\right) \\
\quad+\sum_{\alpha=1}^{N} \int_{t-\left(d_{j} / d_{i}\right) s}^{t} A_{i \alpha} k_{\alpha j}\left(\theta, s-\frac{d_{i}}{d_{j}}(t-\theta)\right) d \theta \\
s<t, k_{i j}(t, s)=\sum_{\alpha=1}^{N} \int_{t-\left(d_{j} / d_{i}\right) s}^{t} A_{i \alpha} k_{\alpha j}\left(\theta, s-\frac{d_{i}}{d_{j}}(t-\theta)\right) d \theta
\end{gathered}
$$


when $i>j$ and $d_{i}<d_{j}$,

$$
\begin{gathered}
s>t, k_{i j}(t, s)=\sum_{\alpha=1}^{N} \int_{0}^{t} A_{i \alpha} k_{\alpha j}\left(\theta, s-\frac{d_{i}}{d_{j}}(t-\theta)\right) d \theta \\
t>s>\frac{d_{i}}{d_{j}} t, k_{i j}(t, s)=\frac{-d_{j}}{d_{j}-d_{i}} \sum_{\alpha=1}^{N}\left(A_{i \alpha}-A_{i \alpha}^{0}\right) J_{\alpha j}\left(\frac{d_{i} t-d_{j} s}{d_{i}-d_{j}}\right) \\
\quad+\sum_{\alpha=1}^{N} \int_{0}^{t} A_{i \alpha} k_{\alpha j}\left(\theta, s-\frac{d_{i}}{d_{j}}(t-\theta)\right) d \theta \\
\frac{d_{i}}{d_{j}} t>s, k_{i j}(t, s)=\sum_{\alpha=1}^{N} \int_{t-\left(d_{j} / d_{i}\right) s}^{t} A_{i \alpha} k_{\alpha j}\left(\theta, s-\frac{d_{i}}{d_{j}}(t-\theta)\right) d \theta ;
\end{gathered}
$$

when $d_{i}=d_{j}$,

$$
\begin{aligned}
& s>t, k_{i j}(t, s)=\sum_{\alpha=1}^{N} \int_{0}^{t} A_{i \alpha} k_{\alpha j}(\theta, s-t+\theta) d \theta \\
& s<t, k_{i j}(t, s)=\sum_{\alpha=1}^{N} \int_{t-s}^{t} A_{i \alpha} k_{\alpha j}(\theta, s-t+\theta) d \theta .
\end{aligned}
$$

Replace $k_{i j}$ by $k_{i j}^{n+1}$ on the left and by $k_{i j}^{n}$ on the right side of all these equations, set $k_{i j}^{0} \equiv 0$ and solve the system iteratively.

If

$$
a=\sup \sum_{\alpha=1}^{N}\left|A_{i \alpha}\right|, \quad b=\sup \frac{d_{j}}{d_{j}-d_{i}}\left|J_{\alpha \beta}\right| \quad \text { for } d_{i} \neq d_{j},
$$

then, by induction, $\left|k_{i j}^{n+1}(t, s)-k_{i j}^{n}(t, s)\right| \leqslant b a^{n+1} t^{n} / n !$, so that

$$
\sum_{n=1}^{\infty}\left(k_{i j}^{n}(t, s)-k_{i j}^{n-1}(t, s)\right)
$$

converges uniformly to a solution $k_{i j}(t, s)$ of the integral equations.

This solution is unique, for if $u_{i j}(t, s)=k_{i j}^{1}-k_{i j}^{2}$, the difference of any two solutions $k_{i j}^{1}$ and $k_{i j}^{2}$ of the integral equations above, then it satisfies the homogeneous equations derived from these by setting $J_{\alpha j}$ to zero.

Let $U(T)=\sup \left|u_{i j}(t, s)\right|$ for all $i, j$ and $t, s \in(0, T)$. Suppose $U\left(T_{1}\right)=0$, where $0 \leqslant T_{1}<T_{0}$ and $U\left(T^{*}\right)>0$ for any $T^{*}>T_{1}$. Since $U\left(T^{*}\right)=\left|u_{i j}(t, s)\right|$ for some $i, j$ and $t, s$ in $\left(T_{1}, T^{*}\right]$ and

$$
u_{i j}(t, s)=\sum_{\alpha=1}^{N} \int_{c}^{t} A_{i \alpha} u_{\alpha j}\left(\theta, s-\frac{d_{i}}{d_{j}}(t-\theta)\right) d \theta,
$$

where $c=$ greater of 0 and $t-\left(d_{j} / d_{i}\right) s$ then it follows that

$$
U\left(T^{*}\right) \leqslant \int_{T_{1}}^{T^{*}} \alpha U\left(T^{*}\right) d \theta=\alpha U\left(T^{*}\right)\left(T^{*}-T_{1}\right)
$$


But $T^{*}$ can be chosen so that $a\left(T^{*}-T_{1}\right)=\frac{1}{2}$ in which case the inequality leads to a contradiction. We must conclude $U\left(T_{0}\right)=0$, and only one solution exists.

Consider now the function $u(x, t)$ defined by equation (2.5). Since $J(0)=I$ and $k^{-}(0, s)=0$, we have

$$
u(x, 0)=v(x, 0)=f(x)
$$

Morenver,

$$
\begin{aligned}
\frac{\partial u}{\partial t}- & D L u-A u=\left(A^{0}-A\right) J_{v}+(J D-D J) L v+D k^{+}(t, 0) D^{-1} f(x) \\
& +\left\{\left[k^{+}(t, t)-k^{-}(t, t)\right]-D\left[k^{+}(t, t)-k^{-}(t, t)\right] D^{-1}\right\} v \\
& -D k^{-}\left(t, T_{0}\right) D^{-1} v\left(x, T_{0}\right) \\
& +\int_{0}^{t}\left(\frac{\partial k^{+}}{\partial t}+D \frac{\partial k^{+}}{\partial s} D^{-1}-A k^{+}\right)(t, \theta) v(x, \theta) d \theta \\
& +\int_{t}^{T_{0}}\left(\frac{\partial k^{-}}{\partial t}+D \frac{\partial k^{-}}{\partial s} D^{-1}-A k^{-}\right)(t, \theta) v(x, \theta) d \theta
\end{aligned}
$$

From the definition of $A^{0}$ it follows that

$$
D A^{0}-A^{0} D=0
$$

and hence if $\phi \equiv D J-J D$, then $\phi=0$ at $t=0$ and

$$
\frac{d \phi}{d t}=D A^{0} J-A^{0} J D=A^{0}(D J-J D)=A^{0} \phi .
$$

Thus

$$
\phi=0 .
$$

Now $k^{-}(0, s)=0$ and the characteristics of the equations for $k^{-}(t, s)$ are lines of the form

$$
s=s_{0}+\left(d_{i} / d_{j}\right)
$$

so that $k^{-}(t, s)=0$ in $s \geqslant \max$ over $i, j$ of $\left(d_{i} / d_{j}\right) t$ and hence $k^{-}\left(t, T_{0}\right)=0$ if $t \leqslant \min$ over $i, j$ of $\left(d_{j} / d_{i}\right) T_{0}$.

These results, together with equations (2.3), (2.4) make the right side of equation 2.7 vanish. Q.E.D.

If $A$ commutes with $D$, then $A=A^{0}$ and $k(t, s)=k^{-}(t, s)=0$. In this case

$$
u=J v, \quad \text { where } \frac{d J}{d t}=A J, \quad J(0)=I .
$$

The operator $J+K$ maps regulated functions $w(t)$ on $\left(0, T_{0}\right)$ into regulated functions on $\left(0, T_{0}\right)$, and at least for $T_{0}$ small enough will have an inverse $J^{-1}-H$ such that $(J+K)\left(J^{-1}-H\right)=I$ or

$$
K J^{-1} w=J H w+K H w .
$$


If we assume $H w$ is of the form

$$
H w=\int_{0}^{t} h^{+}(t, s) w(s) d s+\int_{t}^{T_{0}} h^{-}(t, s) w(s) d s
$$

it will be sufficient to find kernels $h \pm(t, s)$ satisfying the equations

$$
\begin{aligned}
\phi^{+}(t, s) \equiv & k^{+}(t, s) J^{-1}(s)=J(t) h^{+}(t, s)+\int_{0}^{s} k^{+}(t, \theta) h^{-}(\theta, s) d \theta \\
& +\int_{s}^{t} k^{+}(t, \theta) h^{+}(\theta, s) d \theta+\int_{t}^{T_{0}} k^{-}(t, \theta) h^{+}(\theta, s) d \theta, \\
\phi^{-}(t, s) \equiv & k^{-}(t, s) J^{-1}(s)=J(t) h^{-}(t, s)+\int_{0}^{t} k^{+}(t, \theta) h^{-}(\theta, s) d \theta \\
& +\int_{t}^{s} k^{-}(t, \theta) h^{-}(\theta, s) d \theta+\int_{s}^{T_{0}} k^{-}(t, \theta) h^{+}(\theta, s) d \theta,
\end{aligned}
$$

derived from the operator equation (2.8).

THEOREM 2. There exists a unique pair of functions $h^{+}(t, s), h^{-}(t, s)$ which satisfy the hyperbolic equations

$$
\begin{array}{ll}
\frac{\partial h^{+}}{\partial t}+D \frac{\partial h^{+}}{\partial s} D^{-1}+D h^{+} D^{-1} A=0 & \text { in } 0 \leqslant t \leqslant T_{0}, 0 \leqslant s \leqslant t, \\
\frac{\partial h^{-}}{\partial t}+D \frac{\partial h^{-}}{\partial s} D^{-1}+D h^{-} D^{-1} A=0 & \text { in } 0 \leqslant s \leqslant T_{0}, 0 \leqslant t \leqslant s,
\end{array}
$$

and boundary conditions

$$
\begin{gathered}
h^{+}(t, 0)=h^{-}(0, s)=0, \\
{\left[h^{+}(t, t)-h^{-}(t, t)\right]-D\left[h^{+}(t, t)-h^{-}(t, t)\right] D^{-1}=J^{-1}\left(A-A^{0}\right) .}
\end{gathered}
$$

These functions also satisfy equations (2.9), and if $u$ satisfies equations (2.6) in $\left(0, T_{0}\right)$, the function $v=\left(J^{-1}-H\right) u$ satisfies equation (2.1) for $0<t<$ $\min _{i, j}\left(d_{i} / d_{j}\right) T_{0}$.

Proof. Integral equations analogous to those for $k^{ \pm}(t, s)$ may be constructed and solved interatively for $h^{ \pm}(t, s)$ for all $T_{0}$. It is readily shown that both sides of equations (2.9) satisfy the hyperbolic system

$$
\frac{\partial \phi^{ \pm}}{\partial t}+D \frac{\partial \phi^{ \pm}}{\partial s} D^{-1}=A \phi^{ \pm}-D \phi^{ \pm} D^{-1} A^{0}
$$

and boundary conditions

$$
\begin{gathered}
\phi^{+}(t, 0)=\phi^{-}(0, s)=0, \\
{\left[\phi^{+}(t, t)-\phi^{-}(t, t)\right]-D\left[\phi^{+}(t, t)-\phi^{-}(t, t)\right] D^{-1}=A-A^{0} .}
\end{gathered}
$$

This system.has a unique solution. 
In similar fashion we find $v=\left(J^{-1}-H\right) u$ satisfies equation (2.1) if $h^{-}\left(t, T^{0}\right)$ $=0$ and this holds if $0<t<\min _{i, j}\left(d_{i} / d_{j}\right) T_{0}$. Q.E.D.

There is an interesting relationship between equations (2.3), (2.4) for $k^{ \pm}(t, s)$ and equations (2.10), (2.11) for the resolvent kernels $h^{ \pm}(t, s)$. If $A^{\prime}$ denotes the transpose of a matrix $A \in S_{N}$ and $h^{\prime}(t, s)$ denotes the transpose matrix of $h(t, s)$ and if $B=-A^{\prime}, J^{*}=J^{\prime-1}$, then

$$
\begin{array}{cc}
z^{+}(t, s)=-D^{\prime-1} h^{-1}(s, t) D^{\prime} & \text { on } t>s, \\
z^{-}(t, s)=-D^{\prime-1} h^{\prime+}(s, t) D^{\prime} & \text { on } t<s
\end{array}
$$

satisfies the equations

$$
\begin{gathered}
\frac{\partial z^{-}}{\partial t}+D^{\prime} \frac{\partial z^{-}}{\partial s} D^{\prime-1}=-A^{\prime} z^{-}=B z^{-}, \text {on } 0<s<T_{0}, 0<t<s, \\
\frac{\partial z^{+}}{\partial t}+D^{\prime} \frac{\partial z^{+}}{\partial s} D^{\prime-1}=-A^{\prime} z^{+}=B z^{+}, \text {on } 0<t<T_{0}, 0<s<t, \\
z^{-}(0, s)=z^{+}(t, 0)=0, \\
\left(z^{+}(t, t)-z^{-}(t, t)\right)-D^{\prime}\left(z^{+}(t, t)-z^{-}(t, t)\right) D^{\prime-1} \\
=-\left(A^{\prime}-A^{0 \prime}\right) J^{\prime-1}=\left(B-B_{0}\right) J^{*} .
\end{gathered}
$$

Since

$$
\frac{d J}{d t}=A^{0} J, \quad J(0)=I
$$

we have

$$
\frac{d}{d t} J^{\prime-1}=\left(-A^{\prime 0}\right) J^{\prime-1}, \quad J^{\prime-1}(0)=I,
$$

or

$$
\frac{d J^{*}}{d t}=B^{0} J^{*}, \quad J^{*}(0)=I .
$$

Thus for a pair of kernels generated by a matrix $A$, we derive the kernels of its inverse from the system generated by a matrix $B=-A^{\prime}$. Specifically,

$$
h^{+}(t, s)=-D^{-1} z^{\prime-1}(s, t) D, \quad h^{-}(t, s)=-D^{-1} z^{\prime+}(s, t) D .
$$

If $A$ is skew symmetric, then $B=A$ and $z^{ \pm}(t, s)=k^{ \pm}(t, s)$ while $J=I$.

For each bounded matrix $A$ we have operators $J+K$ and $J^{-1}-H$ and to identify the operator with $A$ we can write it as $J_{A}+K_{A}$ etc. Evidently, if

$$
w=\left(J_{B}+K_{B}\right)\left(J_{A}^{-1}-H_{A}\right) u=\left(J^{*}+K^{*}\right) u
$$

and if $u$ satisfies equations (2.6), then $w$ satisfies the same equation with $A$ replaced by $B$. We find that $J^{*}=J_{B} J_{A}^{-1}$ satisfies the system

$$
\frac{d J^{*}}{d t}=B_{0} J^{*}-J^{*} A_{0}, \quad J^{*}(0)=I,
$$


while $k^{* \pm}(t, s)$ satisfy the hyperbolic system

$$
\frac{\partial k^{* \pm}}{\partial t}+D \frac{\partial k^{* \pm}}{\partial s} D^{-1}=B k^{* \pm}-D k^{* \pm} D^{-1} A,
$$

and boundary conditions

$$
\begin{gathered}
k^{*+}(t, 0)=k^{*-}(0, s)=0, \\
{\left[k^{*+}(t, t)-k^{*-}(t, t)\right]-D\left[k^{*+}(t, t)-k^{*-}(t, t)\right] D^{-1}} \\
=\left(B-B^{0}\right) J^{*}-J^{*}\left(A-A^{0}\right) .
\end{gathered}
$$

\section{Kernels for two variable case}

Since the differential operators $\partial / \partial t$ and $\partial / \partial s$ commute with constants, the hyperbolic equation for $k^{+}(t, s)$ and $k^{-}(t, s)$ may, in the case where $A$ and $D$ are constant, be written in the matrix form

$$
\left[A-I \frac{\partial}{\partial t}-\frac{D}{d_{j}} \frac{\partial}{\partial s}\right] k_{j}^{ \pm}=0,
$$

where $k_{j}^{ \pm}$is the $j$ th column of $k^{ \pm}$. If this equation is multiplied by the matrix adjugate operator we see that each element $k_{i j}$ of the vector $k_{j}$ satisfies the differential equation

$$
\left|A-I \frac{\partial}{\partial t}-\frac{D}{d_{j}} \frac{\partial}{\partial s}\right| \phi=0,
$$

where $|B|$ denotes the formal determinant of the matrix $B$.

In the case where $A$ is triangular this differential equation has a simple form:

$$
\begin{aligned}
\left|A-I \frac{\partial}{\partial t}-\frac{D}{d_{j}} \frac{\partial}{\partial s}\right|_{\phi}= & \left(a_{11}-\frac{\partial}{\partial t}-\frac{d_{1}}{d_{j}} \frac{\partial}{\partial s}\right)\left(a_{22}-\frac{\partial}{\partial t}-\frac{d_{2}}{d_{j}} \frac{\partial}{\partial s}\right) \\
& \cdots\left(e_{N N}-\frac{\partial}{\partial t}-\frac{d_{N}}{d_{j}} \frac{\partial}{\partial s}\right) \phi \\
= & 0 .
\end{aligned}
$$

$J$ and the kernels $k^{ \pm}$are also triangular.

In the case $N=2, j=1$, this equation is

$$
\left|\begin{array}{cc}
a_{11}-\frac{\partial}{\partial t}-\frac{\partial}{\partial s} & a_{12} \\
a_{21} & a_{22}-\frac{\partial}{\partial t}-\frac{d_{2}}{d_{1}} \frac{\partial}{\partial s}
\end{array}\right| \phi=0 .
$$


Introduce new variables

$$
\begin{aligned}
& \tau_{1}=t-s, \quad \phi=\psi \exp \left(\frac{a_{22} \tau_{1}+a_{11} x_{1}}{c_{1}}\right), \\
& x_{1}=s-\frac{d_{2} t}{d_{1}}, \quad c_{1}=1-\frac{d_{2}}{d_{1}},
\end{aligned}
$$

and assume $a_{1}>a_{2}>\hat{v}$. Equation (3.3) for $\phi$ gives rise to the equation

$$
\left|\begin{array}{cc}
-c_{1} \frac{\partial}{\partial x_{1}} & a_{12} \\
a_{21} & -c_{1} \frac{\partial}{\partial \tau_{1}}
\end{array}\right| \psi=0
$$

for $\psi$. Since $d_{2} / d_{1}<1, k_{1}^{-}=0$ and hence on $t=s$ or $\tau_{1}=0$,

$$
c_{1} k_{21}^{+}(t, t)=\left(\left(A-A_{0}\right) J_{1}(t)\right)_{21}=a_{21} e^{a_{11} t}, \quad \text { and } \psi_{21}^{+}(x, 0)=a_{21} / c_{1} \text {. }
$$

Thus,

$$
\begin{aligned}
& \frac{\partial^{2}}{\partial \tau_{1} \partial x_{1}} \psi_{21}^{+}=\frac{a_{12} a_{21}}{c_{1}^{2}} \psi_{21}^{+} ; \quad \text { on } \tau_{1}>0, \quad x_{1}>\frac{-d_{2} \tau_{1}}{d_{1}}, \\
& \psi_{21}=0 \quad \text { on } x_{1}=\frac{-d_{2} \tau_{1}}{d_{1}}, \quad \psi_{21}=\frac{a_{21}}{c_{1}} \quad \text { on } \tau_{1}=0 .
\end{aligned}
$$

This implies $\psi_{21}^{+}=0$ in the sector $x_{1}<0$, so that

$$
\psi_{21}=0 \quad \text { on } x_{1}=0, \quad \psi_{21}=\frac{a_{21}}{c_{1}} \quad \text { on } \tau_{1}=0,
$$

and this problem has a similarity solution

$$
\psi_{21}^{+}=\frac{a_{21}}{c_{1}} I_{0}\left(2 \sqrt{\frac{a_{12} a_{21}}{c_{1}^{2}} x_{1} \tau_{1}}\right) .
$$

The first element $k_{11}^{+}$of the vector $k_{1}^{+}$may be found from the relationships

$$
k_{11}^{+}=\psi_{11} \exp \left(\frac{a_{22} \tau_{1}}{c_{1}}+\frac{a_{11} x_{1}}{c_{1}}\right)
$$

where

$$
a_{21} \psi_{11}-c_{1} \frac{\partial \psi_{21}}{\partial \tau_{1}}=0
$$

The case $j=2$ in analogous fashion shows

$$
k_{2}^{+}=0, \quad k_{12}^{-}=\frac{a_{12}}{c_{2}} I_{0}\left(2 \sqrt{\frac{a_{12} a_{21}}{c_{2}^{2}} x_{2} \tau_{2}}\right) \exp \left(\frac{a_{11} \tau_{2}}{c_{2}}+\frac{a_{22} x_{2}}{c_{2}}\right),
$$


where $c_{2}=\left(d_{1} / d_{2}\right)-1, x_{2}=\left(d_{1} / d_{2}\right) t-s, \tau_{2}=s-t$, and

$$
a_{12} k_{22}^{-}+\left(a_{11}-c_{2} \frac{\partial}{\partial \tau_{2}}\right) k_{12}^{-}=0
$$

Thus, for the second order case:

$$
\begin{gathered}
J(t)=\left(\begin{array}{cc}
e^{t a_{11}} & 0 \\
0 & e^{t a_{22}}
\end{array}\right), \\
k^{+}(t, s)=\frac{e^{\alpha_{1} s-\beta_{1} t}}{c_{1}}\left(\begin{array}{cc}
\sqrt{\frac{a_{12} a_{21} x_{1}}{\tau_{1}}} I_{1}\left(2 \sqrt{\frac{a_{12} a_{21}}{c_{1}^{2}} x_{1} \tau_{1}}\right) & 0 \\
a_{21} I_{0}\left(2 \sqrt{\frac{a_{12} a_{21}}{c_{1}^{2}} x_{1} \tau_{1}}\right) & 0
\end{array}\right)
\end{gathered}
$$

where $\alpha_{1}=\left(\left(a_{11}-a_{22}\right) d_{1}\right) /\left(d_{1}-d_{2}\right), \beta_{1}=\left(a_{11} d_{2}-a_{22} d_{1}\right) /\left(d_{1}-d_{2}\right)$,

$$
\begin{aligned}
& k^{-}(t, s)=\frac{e^{\alpha_{2} s-\beta_{2} t}}{c_{2}}\left(\begin{array}{cc}
0 & a_{12} I_{0}\left(2 \sqrt{\frac{a_{12} a_{21}}{c_{2}^{2}} x_{2} \tau_{2}}\right) \\
0 & \sqrt{\frac{a_{12} a_{21} x_{2}}{\tau_{2}}} I_{1}\left(2 \sqrt{\frac{a_{12} a_{21}}{c_{2}^{2}} x_{2} \tau_{2}}\right)
\end{array}\right) \\
& \text { where } \alpha_{2}=\left(\left(a_{11}-a_{22}\right) d_{2}\right) /\left(d_{1}-d_{2}\right), \beta_{2}=\beta_{1} \text {. }
\end{aligned}
$$

The kernels $h^{ \pm}(t, s)$ for the resolvent kernels of $K$ may be constructed from its adjoint properties derived in Section 2.

For the present case $N=2$ and constant $A$ this mapping (2.12) gives

$$
\begin{aligned}
& h^{+}(t, s)=\frac{-e^{-\alpha_{2} t+\beta_{2} s}}{c_{2}}\left(-\frac{d_{1}}{d_{2}} a_{21} I_{0}\left(2 \sqrt{\frac{a_{12} a_{21}}{c_{2}^{2}} \bar{x}_{2} \bar{\tau}_{2}}\right) ;\right. \\
& \left.\times \sqrt{\frac{a_{12} a_{21}}{\bar{\tau}_{2}}} I_{1}\left(2 \sqrt{\frac{a_{12} a_{21}}{c_{2}^{2}} \bar{x}_{2} \bar{\tau}_{2}}\right)\right) \\
& h^{-}(t, s)=\frac{-e^{-\alpha_{1} t+\beta_{1} s}}{c_{1}}\left(\sqrt{\frac{a_{12} a_{21} \bar{x}_{1}}{\bar{\tau}_{1}}} I_{1}\left(\underset{0}{2 \sqrt{\frac{a_{12} a_{21}}{c_{1}^{2}} \bar{x}_{1} \bar{\tau}_{1}}}\right)\right. \\
& \left.-\frac{d_{2}}{d_{1}} a_{12} I_{0}\left(2 \sqrt{\frac{a_{12} a_{21}}{c_{1}^{2}} \bar{x}_{1} \bar{\tau}_{1}}\right)\right)
\end{aligned}
$$


where $\bar{x}_{1}=t-\left(d_{2} / d_{1}\right) s, \bar{\tau}_{1}=s-t, \bar{x}_{2}=\left(d_{1} / d_{2}\right) s-t, \bar{\tau}_{2}=t-s$. This agrees with the expression derived by Hill [3].

The function

$$
k(t, s)= \begin{cases}k^{+}(t, s) & \text { for } t>s, \\ k^{-}(t, s) & \text { for } t<s\end{cases}
$$

is bounded by $b a e^{a T}$ for $i<T, s<T$ (where $a$ and $b$ are the sup norms for $K$ and $J$ used in Section 2) and is discontinuous at only a finite set of points on any line $s=s_{0}>0, t=t_{0}>0$. It therefore has a double Laplace Transform:

$$
\tilde{\bar{k}}=\int_{0}^{\infty} e^{-p t} \int_{0}^{\infty} e^{-q s} k(t, s) d t d s, \quad p>a, q>a,
$$

and

$$
A \tilde{\bar{k}}=\int_{0}^{\infty} \int_{0}^{\infty} e^{-p t-q s}\left(\frac{\partial k}{\partial t}+D \frac{\partial k}{\partial s} D^{-1}\right) d t d s
$$

so that

$$
\begin{aligned}
A \tilde{\bar{k}}-p \tilde{\bar{k}}-q \tilde{\bar{k}} D^{-1} & =\int_{0}^{\infty} \int_{0}^{\infty} \frac{\partial}{\partial t}\left[e^{-p t-q s} k\right]+\frac{\partial}{\partial s}\left[D e^{-p t-q s} k D^{-1}\right] d t d s \\
& =\int_{s=t} e^{-p t-q s}\left[k^{+}-k^{-}\right] d s-D e^{-p t-q s}\left(k^{+}-k^{-}\right) D^{-1} d t \\
& =\int_{0}^{\infty} e^{-p t-q t}\left(A-A^{0}\right) J(t) d t \\
& =\left(A-A^{0}\right)\left[(p+q) I-A^{0}\right]^{-1} .
\end{aligned}
$$

If $\bar{k}_{j}$ denotes the single Laplace Transform $\int_{0}^{\infty} e^{-q s} k_{j}(t, s) d s$, then we find from the expression for $\tilde{\bar{k}}_{j}$ that

$$
\bar{k}_{j}=e^{t\left(a_{j}-q\right)}\left\{e^{t\left[A-A^{0}-q\left(D_{j}-I\right)\right]}-I\right\}\left\{A-A^{0}-q\left(D_{j}-I\right)\right\}^{-1}\left(A-A^{0}\right)_{j}
$$

in the case $A^{0}$ diagonal. $a_{j}$ is the $j$ th diagonal element of $A^{0}$ and $D_{j}=D / d_{j}$.

The transform $\vec{k}_{j}$ may be obtained in another way as follows. The operator $L=-q I$ commutes with $A$ and $D$ and the problems

$$
\frac{\partial u}{\partial t}=-D q u+A u, \quad u(0)=I, \quad \frac{d v}{d t}=-D q v, \quad v(0)=I,
$$

have solutions $u=e^{t(A-D q)}$ and $e^{-t D q}$.

The relationship

$$
u=J v+k v
$$

gives the result

$$
e^{t(A-D q)}=e^{t A_{0}} e^{-t D q}+\int_{0}^{t} k^{+}(t, s) e^{-s D q} d s+\int_{t}^{T_{0}} k^{-}(t, s) e^{-s D q} d s,
$$


and hence considering the $j$ th column only we have

$$
\int_{0}^{\infty} k_{j}(t, s) e^{-s q^{*}} d s=\left[e^{t\left[A-D_{j} q^{*}\right]}-e^{l\left[A_{0}-q^{*} D_{j}\right]}\right]_{j}
$$

where $q^{*}=q d_{j}, D_{j}=D / d_{j}$ and $[B]_{j}$ denotes the $j$ th column of $B$.

\section{Nonhomogeneous problems}

When $D$ and $A$ commute, the solutions of the nonhomogeneous problem

$$
\frac{\partial u}{\partial t}=D L u+A u+\phi(x, t), \quad u(x, 0)=f(x),
$$

can be expressed in the form

$$
u=J v, \quad \text { where } \frac{d J}{d t}=A J, \quad J(0)=I,
$$

where $v$ satisfies the inhomogeneous problem

$$
\frac{\partial v}{\partial t}=D L v+\psi(x, t), \quad \psi(x, t)=J^{-1} \phi(x, t), \quad v(x, 0)=f(x) .
$$

This result extends to the noncommuting case where $D, L$, and $A$ have the properties required in Theorem 1.

THEOREM 3. If $v(x, t)$ satisfies the equation

$$
\frac{\partial v}{\partial t}(x, t)=D L v(x, t)+\psi(x, t), \quad v(x, 0)=f(x),
$$

and $J(t), k^{ \pm}(t, s)$ satisfy equations (2.2), (2.3), (2.4), then

$$
u(x, t)=(J+K) v(x, t)
$$

satisfies the equations

$$
\frac{\partial u}{\partial t}=D L u+A u+\phi
$$

where

$$
\phi(x, t)=D(J+K) D^{-1} \psi(x, t) .
$$


Proof. From equation (4.4) we have

$$
\begin{aligned}
\frac{\partial u}{\partial t}-D L u-A u= & \left(-A+A^{0}\right) J v+J\left(\frac{\partial v}{\partial t}-D L v\right) \\
& +\left[k^{+}(t, t)-k^{-}(t, t)\right] v+\int_{0}^{t} \frac{\partial k^{+}}{\partial t} v \\
& +\int_{t}^{T_{\mathrm{v}}} \frac{\partial k^{-}}{\partial t} v-\int_{0}^{t} A k^{+} v-\int_{t}^{T_{0}} A k^{-} v-\int_{0}^{t} D k^{+} D^{-1}\left(\frac{\partial v}{\partial t}-\psi\right) \\
& -\int_{t}^{T_{0}} D k^{-} D^{-1}\left(\frac{\partial v}{\partial t}-\psi\right) \\
= & J \psi+\int_{0}^{t} D k^{+} D^{-1} \psi+\int_{t}^{t_{0}} D k^{-} D^{-1} \psi \\
= & D(J+K) D^{-1} \psi \\
= & \phi . \quad Q . E . D .
\end{aligned}
$$

It can be shown in analogous fashion if $u$ satisfies equation (4.5) and $v$ is defined by

$$
v+\left(J^{-1}-H\right) u
$$

where $J^{-}-H$ is the inverse of $J+K$, then $v$ satisfies equation (4.3) where

$$
\psi=D\left(J^{-1}-H\right) D^{-1} \phi . \quad \text { Q.E.D. }
$$

\section{References}

[1] J. Dieudonné, Foundations of modern analysis (Academic Press, New York, 1962).

[2] P. Garabedian, Partial differential equations (Wiley, New York, 1964).

[3] J. M. Hill, "On the solution of reaction-diffusion equations", IMA J. Appl. Math. 27 (1981), 177-199. 